\title{
Corrigendum to "A New Method of Scanning Tunneling Spectroscopy for Study of the Energy Structure of Semiconductors and Free Electron Gas in Metals"
}

\author{
A. Pavlov and H. Ihantola \\ Laboratory of Electronics and Information Technology, University of Turku, Turku, Finland \\ Correspondence should be addressed to A. Pavlov; pavlov211@outlook.com
}

Received 4 January 2021; Accepted 4 January 2021; Published 23 January 2021

Copyright (C) 2021 A. Pavlov and H. Ihantola. This is an open access article distributed under the Creative Commons Attribution License, which permits unrestricted use, distribution, and reproduction in any medium, provided the original work is properly cited.

In the article titled "A New Method of Scanning Tunneling Spectroscopy for Study of the Energy Structure of Semiconductors and Free Electron Gas in Metals" [1], the authors identified an error in Equation (3), which should be corrected as follows:

Original text

$$
I_{t} \propto \frac{1}{l}\left(E_{g n} \exp \left(\xi \frac{e V-e V_{S P V}}{E_{g n}}\right)-E_{g p} \exp \left(\xi \frac{-e V-e V_{S P V}}{E_{g p}}\right)\right)
$$

The corrected formula (3):

$I_{t} \propto \frac{1}{l}\left(E_{g n} \exp \left(\xi \frac{e V-e V_{S P V}}{E_{g n}}\right)-E_{g p} \exp \left(\xi \frac{-\left(e V-e V_{S P V}\right)}{E_{g p}}\right)\right)$

\section{References}

[1] A. Pavlov and H. Ihantola, "A New Method of Scanning Tunneling Spectroscopy for Study of the Energy Structure of Semiconductors and Free Electron Gas in Metals," The Journal of Scanning Microscopies, vol. 19, no. 7, pp. 459-465, 2006. 